\section{MedienPädagogik}

www. medienpaed.com
Zeitschrift für

Theorie und Praxis

der Medienbildung

ISSN 1424-3636

Themenheft Nr. 15/16: Computerspiele und Videogames

in formellen und informellen Bildungskontexten

\title{
Unterrichten mit Computerspielen \\ Didaktische Potenziale und Ansätze für den gezielten Einsatz in Schule und Ausbildung
}

Dominik Petko

\begin{abstract}
Der besondere Wert von Computerspielen und Videogames für informelle und formelle Lernprozesse wird in den letzten Jahren intensiv diskutiert. Für schulische Kontexte ist es von besonderer Bedeutung, nicht nur die allgemeinen Potenziale solcher Spiele zu erkennen, sondern sie auch mit geeigneten Unterrichtsarrangements umzusetzen. Der Artikel gibt einen Überblick über die grundlegenden Konzepte und Ansätze, die dabei für die schulische Praxis relevant sein können.
\end{abstract}

Seit einigen Jahren scheinen die Fronten in den Publikationen zum Themenkreis des Aufwachsens mit Video- und Computerspielen festgefahren. In immer schrilleren Tönen wird entweder auf die besonderen Potenziale oder aber auf die besonderen Gefahren von digitalen Spielen hingewiesen. Befürworterinnen und Befürworter sehen in Computer- und Videospielen nahezu idealtypisch eine Form des aktiven, selbstgesteuerten, konstruktiven, motivierten, situierten und sozialen Lernens (z. B. Kutner \& Olson, 2008; Johnson, 2005; Aldrich, 2004; Gee, 2003; Prensky, 2001). Für Gegnerinnen und Gegner bedeuten digitale Games dagegen vor allem Zeitverschwendung, Vereinsamung, Bewegungsarmut, Gewaltverherrlichung, Computerspielsucht oder geistige Verarmung (z. B. Bauerlein, 2008; Pfeiffer, Mössle, Kleimann \& Rehbein, 2007; Spitzer, 2005). Problematische Aspekte und positive Potenziale bilden, wenn sie nicht in populärwissenschaftlicher Überspitzung aufeinander treffen, nicht notwendigerweise einen Widerspruch, sondern zeigen unter Umständen die tatsächliche Ambivalenz des Themas (vgl. z. B. Lee \& Peng, 2006; Klimmt, 2004). Lehrpersonen wird es gegenwärtig jedoch leichter gemacht, sich pauschal den Gegnerinnen und Gegnern des Lernens mit digitalen Games anzuschliessen, da diese mit Vermeidung und Verbot die einfacheren Rezepte zur Umsetzung ihrer Ansichten in der Hand haben. Begeisterte und vorsichtige Befürworterinnen und Befürworter befinden sich demgegenüber in einer schwierigen Bringschuld. Sie müssen zeigen, dass digitale Spiele nicht nur theoretisch ein Potenzial besitzen, sondern im Unterricht mit vertretbarem Aufwand und begründbaren Mehrwerten einsetzbar sind.

Damit die Potenziale des Lernens mit Computer- und Videospielen auch in Schulen zum Thema werden, ist eine Differenzierung der allzu allgemein geführten Diskussion nötig. Dabei müssen informelle Lernprozesse, die sich beim Spielen 
von Games in der Freizeit ergeben, von formellen Lernprozessen unterschieden werden, die beim gezielten schulischen Einsatz von Computerspielen zum Tragen kommen. Eine weitere wichtige Unterscheidung ist die Frage, ob es sich bei den eingesetzten Spielen um unterhaltungsbezogene Computerspiele oder um spezifische Lernspiele handelt, was jedoch nur der erste Schritt für genauere Analyse und Typisierung von Spielen sein muss. Die für die Unterrichtspraxis wichtigste Ebene fragt schliesslich nach den eingesetzten Strategien und Unterrichtsarrangements der Lehrenden und deren Angemessenheit für Lernende in Bezug auf bestimmte Lernziele. Die folgende Darstellung versucht einige Ansätze zu beschreiben, mit denen das Lehren mit Computerspielen an den allgemeindidaktischen Diskurs anschlussfähig und damit für Schule und Unterricht attraktiv werden kann. Möglichkeiten für eine sinnvolle Einbettung in den Unterricht werden skizziert.

\section{Ansätze zur gezielten Nutzung}

In der theoretischen Literatur finden sich eine ganze Reihe systematischer Überblicksdarstellungen der allgemeinen Potenziale, die digitale Spiele als Lern- und Unterrichtsmedien als besonders geeignet erscheinen lassen (z. B. Susi, Johannesson \& Backlung, 2007; Gebel, 2006; Mitchell \& Savill-Smith, 2004; Kirriemuir \& McFarlane, 2003; Squire, 2003). Deutlich weniger Darstellungen beschäftigen sich dagegen mit den konkreteren Fragen des Einsatzes von Videogames im Unterricht (z. B. Van Eck, 2006; Sandford \& Williamson, 2005; Egenfeldt-Nielsen, 2004; Schrackmann, Knüsel, Moser, Mitzlaff \& Petko, 2008). Zwar ist es wahrscheinlich, dass auch die unsystematische Beschäftigung mit digitalen Spielen ein implizites Lernen verschiedener Fähigkeiten ermöglicht, dafür haben Lehrpersonen in Schulen angesichts enger Lehrpläne und grosser Heterogenität der Schülerschaft jedoch weder den Auftrag noch die Zeit. Der Einsatz von Videospielen steht in Konkurrenz zum Einsatz anderer Medien (z. B. einfacher Arbeitsblätter) und muss in diesem Vergleich sowohl handhabbar sein als auch besondere Mehrwerte besitzen. Auch für engagierte Lehrpersonen können die besonderen Potenziale erst dann Plausibilität gewinnen, wenn sie auf Unterrichtssituationen hin konkretisiert werden. Grundansätze für eine solche mediendidaktische Konkretisierung werden hier im Überblick dargestellt.

\section{Strategisches Problemlösen vermitteln}

Spiel kann allgemein als eine nicht-zweckrationale Aktivität mit Vergnügungscharakter charakterisiert werden, die eine von der Alltagswelt unterschiedene Eigengesetzlichkeit besitzt. Spielen, sowohl in Form des freien als auch des regelgeleiteten Spiels, stellt trotz seines Spass-Charakters durch probehaftes Problemlösen und soziales Rollenspiel eine wichtige Form lebenslangen Lernens dar (vgl. Flitner, 2004; Oerter, 1999). Problemlösen als ein Ansatz des «learning by doing» wurde in der Tradition von John Dewey und anderen auch als wichtiges Lernarrangement 
der Schule begründet und vielfach dem rezeptiven Lernen gegenüber als überlegen betrachtet, obwohl der Nachweis der besonderen didaktischen Wirksamkeit letztlich schwierig blieb (vgl. Preckel, 2004; Dochy, Segers, Van den Bossche \& Gijbels, 2003). Mittlerweile plädieren verschiedene Autorinnen und Autoren entlang einer "gemässigt konstruktivistischen Position» für einen sinnvollen Wechsel von entdeckenden, rezipierenden, übenden und anwendenden Lernphasen (vgl. z. B. Reinmann-Rothmeier \& Mandl, 2006).

Im Unterschied zum nicht-medialen Spiel geschieht das Problemlösen in Computer- und Videospielen in einem stärker durch die Software vorstrukturierten Raum. Spielerinnen und Spieler stehen dabei in der Mehrheit der Spiele vor der Aufgabe, einen suboptimalen Anfangszustand in einen optimalen Endzustand zu überführen. Dabei muss ein komplexes Zusammenspiel von unterschiedlichen Variablen beachtet werden, von denen sich einige über die Gamecontroller (d. h. Tastatur, Mouse, Gamepad, Joystick etc.) rundenbasiert oder in Echtzeit beeinflussen lassen. Indem der Computer auf die Inputs des Spielenden entlang bestimmter Algorithmen reagiert, entsteht eine Interaktion zwischen den Problemlösungsversuchen der bzw. des Spielenden und dem Feedback des Computers. Im Idealfall gewinnt dies den Charakter eines Problemlösezirkels, der vom Bemerken und Analysieren einer Schwierigkeit, über das Entwickeln und Abwägen von Lösungsvarianten zu einem Einsatz und einer Evaluation des Resultats führt, bevor er allenfalls von neuem beginnt (vgl. im Überblick: Reusser, 2005). Dennoch ist nicht zu erwarten, dass der Einsatz digitaler Spiele automatisch zu verbesserten Problemlösestrategien führt. Gründe und mögliche Massnahmen lassen sich aus den Erfahrungen zu computerbasierten Simulationen gewinnen (vgl. de Jong \& van Joolingen, 1998). So werden etwa keine systematischen Hypothesen aufgestellt, zu viele Variablen auf einmal variiert, die Resultate ungenau beobachtet und die weiterführende Strategie nicht konsequent an den bisherigen Resultaten angepasst. Lehrpersonen können solchen Schwierigkeiten durch vorstrukturierende Hinweise oder durch situatives Coaching begegnen.

Wenn Computerspiele im Unterricht gezielt zur Vermittlung von Problemlösestrategien eingesetzt werden sollen, dann kann dies sowohl mit unterhaltungsbezogenen Spielen als auch mit Lernspielen geschehen. Bei unterhaltungsorientierten Spielen dürfte es jedoch nötig sein, bestimmte prototypische Problemsituationen auszuwählen und Schülerinnen anzuregen, diese systematisch anzugehen. Für den Unterricht ist es hilfreich, wenn die gewählten Spiele rundenbasiert ablaufen oder über eine Pause-Funktion besitzen. Insbesondere wenn in Arbeitsgruppen gearbeitet wird, dann bietet sich damit eine gute Möglichkeit, das Spiel anzuhalten und sich gemeinsam eine Strategie für die nächste Spielphase zu überlegen. 


\section{Situiertes Lernen ermöglichen}

Die grosse Mehrheit digitaler Spiele hat einen erzählerischen Spielsinn oder eine sich narrativ entwickelnde Spielhandlung. Die Spiel-Storys können mehr oder weniger rudimentär oder ausführlich, realitätsbezogen oder fiktiv sein. Mit der Theorie des «situated learning» gilt mittlerweile als erwiesen, dass Lernen mit Vorteil in sinnvollen Kontexten geschieht und dass ein abstraktes Lernen schwerwiegende Transferprobleme mit sich bringt (vgl. Lave \& Wenger, 1991; Collins, Brown \& Newman, 1989). Auf diesem Hintergrund entstand der Ansatz der «anchored instruction», der zeigte, dass sich Lernprozesse nicht nur in lebensweltlichen Kontexten, sondern auch mit elektronischen Medien situieren lassen (Bransford, Sherwood, Hasselbring, Kinzer \& Williams, 1990). Im wegweisenenden Jasper-Projekt lösen Kinder mathematische Aufgaben eingebettet in die filmischen Abenteuer des Jasper Woodbury, was zum Vorbild für viele andere narrative Lernspiele wurde (vgl. Cognition and Technology Group at Vanderbilt, 1997).

Die Potenziale digitaler Spielhandlungen können jedoch noch weit über die Situierungsfunktion hinausgehen. Spiele wecken das Interesse für bestimmte Inhaltsbereiche oder können sogar ein diesbezügliches Wissen vermitteln. Letzteres kann natürlich nur dann relevant sein, wenn die Handlung einen ausreichenden Realitätsgehalt besitzt oder in ihrer Fiktionalität eine Reflexion realen Wissens ermöglicht. Der Realitätsbezug von Videospielen kann auf unterschiedlichen Ebenen liegen. Thematischen Realitätsbezug besitzen Spiele, die einen echten kulturellen oder naturwissenschaftlichen Sachverhalt möglichst korrekt repräsentieren (z. B. das Leben in einer bestimmten Epoche, das Fahrverhalten eines Autos). Da eine grosse Zahl handelsüblicher Spiele in fiktiven Welten angesiedelt ist oder die Darstellung realer Sachverhalte dramatisch überspitzt wird, sind vergleichende oder reflektierende Arbeitsaufträge für die Nutzung dieser Spiele zentral. So lässt sich beispielsweise aus historisch orientierten Strategiespielen einiges über Imperialismus lernen, insbesondere wenn man die Logik des Spiels und die der historischen Vorbilder vergleicht. Logisch-mathematischen Realitätsbezug besitzen Spiele, in denen Abstraktions- und Rechenleistungen gefragt sind, die denen in Schule und Alltag möglichst nahe kommen (z. B. das Schätzen von Einflussfaktoren, das Kalkulieren von Anteilen). In Strategiespielen lassen sich beispielsweise Entscheide und ihre Auswirkungen durch die Erstellung von Datenreihen und Kurven mathematisch besser verstehen. Sozialen Realitätsbezug haben Spiele, in denen soziale Konstellationen simuliert oder herbeigeführt werden, die im realen Leben in ähnlicher Form vorkommen (z. B. Varianten der Arbeitsteilung oder der Zusammenarbeit). Netzwerkspielen bieten viele Anlässe zur Reflexion des sozialen Dilemmas zwischen Kooperation und Konkurrenz. Spielehandlungen lassen sich wie Texte oder Filme «lesen» und analysieren (vgl. Ammann \& Hermann, 2004). Narrative Handlungen haben darüber hinaus emotionale Qualitäten, deren Bedeutung für Lernprozesse erst langsam in den Blickpunkt der Aufmerksamkeit rückt. In fremd- 
sprachigen Spielen liegen schliesslich grosse Potenziale für den Fremdsprachenunterricht, insbesondere, wenn das Spiel Dialogsituationen ermöglicht. Für eine gezielte didaktische Nutzung der narrativen Potenziale von Videospielen im Unterricht oder in Seminarkontexten stellen sich hier einige ganz praktische Probleme. Insbesondere kommerzielle Spiele haben eine Spielhandlung, die nicht selten mehr als 20 Stunden Spielzeit umfasst. Da ein volles Durchspielen innerhalb der Unterrichtszeit auch in Projektwochen nicht möglich ist, bietet es sich an, nur mit einzelnen Episoden zu arbeiten. Vorausgehende Handlungen lassen sich erzählen oder als Text lesen. Bedingung für dieses Vorgehen ist natürlich, dass das Spiel Speicherstände zulässt und diese sich problemlos auf die Unterrichtscomputer kopieren lassen. Lehrpersonen kommen jedoch kaum darum herum, das Spiel bis zu diesem Stand selbst zu spielen und sich Gedanken über die Einsatzmöglichkeiten zu machen.

\section{Komplexe Zusammenhänge veranschaulichen}

Im Sinne von Simulationen mehr oder weniger realitätsnaher Zusammenhänge kombinieren digitale Spiele Informationen in vielfältiger Modalität (d. h. sie sprechen verschiedene Sinneskanäle an) und Codalität (d.h. sie verwenden dabei unterschiedliche Symbolsysteme; vgl. Weidenmann, 2002). Die Wirksamkeit solch reichhaltiger Präsentationen für das Erlernen komplexer Zusammenhänge kann als erwiesen gelten, wenn sie die Lernenden mit ihrer Fülle nicht überfordern und Elemente optimal kombinieren (vgl. z. B. Petko \& Reusser, 2005; Schnotz, 2002; Mayer, 2001). Für Schule und Ausbildungskontexte gelten solche Potenziale vor allem für Spiele mit einem ausreichenden Realitätsgehalt. Insbesondere wenn mit unterhaltungsorientierten Videospielen gearbeitet wird, kann das Geschehen auf dem Bildschirm oder die Schwierigkeit der Steuerung aber auch von wesentlicheren Aspekten ablenken. Ausgehend von Theorien des «cognitive load» sollte möglichst viel kognitive Kapazität auf lernrelevante Aspekte und möglichst wenig auf Nebensächliches verwendet werden (vgl. Chandler \& Sweller, 1991). Dies kann einerseits durch die Auswahl eines geeigneten Spiels gewährleistet werden oder durch besondere Beobachtungsaufträge kompensiert werden, die die Aufmerksamkeit auf die inhaltlich relevanten Aspekte lenken. Aktivierende und fokussierende Arbeitsaufträge erscheinen aber auch noch aus einem anderen Grund als sinnvoll. Wie aus den Arbeiten von Salomon $(1984 ; 1983)$ zur Nutzung von Videomedien als Unterrichtsgegenstand bekannt ist, besteht die Gefahr, dass die Aufmerksamkeit von Lernenden gegenüber dem vermeintlich «leichten Medium» sinkt. Das Ausmass der eingesetzten kognitiven Aktivität («amount of invested mental effort») muss gezielt gefördert werden, wenn der Einsatz des Mediums für Lernzwecke nicht mit einer reinen unterhaltungsbezogenen Aktivität verwechselt werden soll. Höchstwahrscheinlich ist das bei Videospielen noch verstärkt der Fall. Die Begeisterung, im Unterricht «spielen» zu können, muss mit einem Fokus auf 
das intendierte «Lernen» ergänzt werden. Im Unterricht bieten sich hier viele aus der Filmbildung bereits bewährte Ansätze. Wenn in Gruppen an einem Videospiel gearbeitet wird, dann lassen sich z. B. unterschiedliche Rollen verteilen, so dass sich verschiedene Schüler/innen auf unterschiedliche Aspekte konzentrieren, während eine/r das Spiel steuert. Die Notizen können nachher zusammengetragen und weiter im Unterricht thematisiert werden.

\section{Lernprozesse motivieren}

Eine grosse Mehrheit der männlichen Jugendlichen und eine wachsende Zahl weiblicher Jugendlicher spielen regelmässig und freiwillig in ihrer Freizeit Video- und Computerspiele (vgl. Feierabend \& Rathgeb, 2007b, 2007a). Warum Computerspiele ein dermassen offensichtliches und grosses Motivationspotenzial besitzen, wurde bereits eingehend theoretisch beleuchtet (vgl. z. B. Wünsch \& Jenderek, 2008; Klimmt \& Hartmann, 2006; Fritz, 2003, 1995). Zusammenfassend ist zu vermuten, dass Computerspiele den Bedingungsfaktoren intrinsischer Motiviertheit nahezu idealtypisch entsprechen. Nach Deci \& Ryan (1993; 1985) steht intrinsische Motivation in einem engen Zusammenhang mit den Grundbedürfnissen des Autonomieerlebens, Kompetenzerlebens und der sozialen Eingebundenheit.

Autonomieerleben wird in digitalen Spielen durch ihre einfache Verfügbarkeit und die Wahlmöglichkeiten innerhalb des Spiels gefördert. Im Unterschied zu Filmen und Videos ist der Spieler bzw. die Spielerin aktiv und schlüpft nicht selten in prestigeträchtige Rollen von z. B. Held(inn)en, Pilot(inn)en oder anderen Fantasiefiguren, deren Aufgaben Handlungsfreiheit suggerieren, auch wenn die Aktionsmöglichkeiten innerhalb des Games mehr oder weniger beschränkt sind. Die adaptive Machart von digitalen Spielen ermöglicht kalkulierbare Kompetenzerlebnisse mit schnellem Feedback und nahezu optimalem Anstieg der Schwierigkeit. Ranks und andere Leistungsinsignien erlauben in vielen Spielen den Aufbau dauerhafter Zeichen der eigenen Fähigkeiten, so dass bereits das Starten des Spiels und der Wiedereinstieg auf hohem Niveau das Erleben von Selbstwirksamkeit fördert.

Das Bedürfnis nach sozialer Eingebundenheit kann in Videospielen schliesslich entweder durch parasoziale Interaktion mit Spielfiguren oder durch die Beteiligung an Online-Communitys erfüllt werden. Auf diese Weise können Videospiele auch verstärkt als Instrument des Mood-Managements in den Blick genommen werden. Im Spiel kann das Zusammenspiel von Stress, Aktion und erfolgreichem Feedback zu einem Zustand des Flow-Erlebens führen, in dem Zeit und Umwelt vergessen wird (vgl. Czíkszentmihályi, 1990). Dies erstaunt vor allem bei Kindern, denen im schulischen Kontext kaum je derartige Aufmerksamkeit, Konzentration oder Begeisterungsfähigkeit zuzutrauen wäre. Bei exzessiver Nutzung häufen sich damit auch Befunde, die digitalen Spielen ein ernstzunehmendes Suchtpotenzial bescheinigen (vgl. Grüsser \& Thalemann, 2006). Für schulischen Einsatz steht jedoch das erstaunliche Motivationspotenzial im Vordergrund, das eine Involvie- 
rung von Schülerinnen und Schülern erlaubt, die ansonsten in dieser Form nicht zu motivieren wären. Dabei ist jedoch zu erwarten, dass die motivationsförderlichen Potenziale noch einen anderen Charakter gewinnen als in Freizeitkontexten. Autonomieerleben und Kompetenzerleben entsteht hier auch durch die Möglichkeit des eigenständigen Arbeitens und den Umstand, dass spielgewohnte Schülerinnen und Schüler in eine Expert(inn)en-Rolle schlüpfen können, auch gegenüber der Lehrperson. Der Faktor der sozialen Eingebundenheit entsteht im Klassenkontext vor allem dann, wenn in Gruppen gearbeitet wird, in denen bestimmte Produkte erstellt werden müssen, wobei nach Möglichkeit zugleich Einzel- und Teamleistungen gewürdigt werden und damit eine positive Interdependenz innerhalb der Gruppe entsteht (Johnson \& Johnson, 1998; Slavin, 1996).

\section{Soziales Lernen fördern}

Das gemeinsame Spielen von digitalen Games, online oder in der Präsenzgruppe, erlaubt ein vielfältiges soziales Lernen, das jedoch noch verhältnismässig wenig untersucht ist. Dabei dürften insbesondere bei komplexen Aufgaben, die eine hohe Koordination erforderlich machen, praktisch alle bekannten Potenziale sozialen Lernens zum Tragen kommen (vgl. Fischer, 2001; Slavin, 1996). Im gemeinsamen Austausch wird spielrelevantes Wissen ausgetauscht, ergänzt, strukturiert, hinterfragt, diskutiert und vergewissert. Dies geschieht mehr oder weniger explizit in ganz unterschiedlichen Kanälen - enaktiv im Medium des Spiels, aber auch in spielbegleitenden Chats und Audiokonferenzen, in Foren auf Websites der Spieler-Community oder im Gespräch im Freundeskreis. An LAN-Events und ESports-Veranstaltungen treffen sich virtuelle Communitys oder die stärker formalisierten Clans auch in der realen Welt, wobei ebenfalls die soziale Dimension im Vordergrund steht (vgl. Hepp \& Vogelgesang, 2008; Jansz \& Martens, 2005). Die These, dass Computerspielende sozial verarmt sind, kann kaum noch aufrechterhalten werden. Stattdessen muss angenommen werden, dass es insbesondere bei männlichen Jugendlichen heute innerhalb der Peer-Group ein Stigma sein kann, sich nicht mit Computerspielen zu beschäftigen. Insbesondere in Online-Spielen geht es auch um Teamwork, Fairness und Rollenkompetenzen. In Anlehnung an gängige Theorien der Identitätskonstruktion kann es in der Steuerung virtueller Spielfiguren auch um Rollendistanz, Ambiguitätstoleranz und Frustrationstoleranz gehen. Die in digitalen Spielen erworbenen Kompetenzen ähneln den Erfordernissen der gegenwärtigen Arbeitswelt, in virtuellen Teams zusammenzuarbeiten. Erst jüngst veröffentlichte IBM eine Studie mit der Empfehlung, Erfahrungen in der Leitung von Clans oder E-Events in Lebensläufen für Bewerbungszwecke zu erwähnen (vgl. Reeves, Malone \& al., 2007).

Im schulischen Einsatz fördern Computerspiele ein soziales Lernen im Klassenzimmer vor allem durch Gruppenarbeit, die schon aus praktischen Gründen kaum umgänglich ist. In den wenigsten Klassenzimmern herrscht eine Vollausstattung mit 
Computern und spezialisierte Computerräume sind vor allem in höheren Schulstufen zu finden (vgl. Barras \& Petko, 2007; Krützer \& Probst, 2006). Der Einsatz von Computerspielen erfordert damit vor allem auf frühen Schulstufen eine Binnendifferenzierung, in der Gruppen von Schülern/-innen bzw. einzelne an unterschiedlichen Aufgaben arbeiten. Die Arbeit am Computerspiel kann auch im Rahmen von Stationenarbeit oder im Wochenplan flexibilisiert werden. Die Gruppendynamik bei der Arbeit am Computerspiel gestaltet sich anders, je nachdem, ob in leistungshomogenen oder leistungsheterogenen Gruppen zusammengearbeitet wird. Bei beiden Varianten macht es Sinn, ein bestimmtes Kooperationsskript zur Orientierung vorzugeben oder eine Planung von Aufgaben- und Rollenverteilungen als expliziten Arbeitsschritt den Schülerinnen und Schülern zu überlassen. Dabei sollte vor allem darauf geachtet werden, dass jeder Schüler bzw. jede Schülerin eine sinnvolle Aufgabe im Team hat und niemand einfach «danebensitzt». In leistungsheterogenen Gruppen ergeben sich besondere Potenziale dadurch, wenn leistungsschwächere Lernende den Lead haben bzw. das Spiel steuern können und leistungsstärkere Coachingfunktionen übernehmen, in denen sie z. B. nur dann helfen, wenn andere nicht weiterkommen. Ausserdem macht es Sinn, wenn nicht nur kooperative (d. h. aufteilende), sondern auch kollaborative (d. h. gemeinsame und konvergenzstiftende) Arbeitsschritte gefragt sind. Lehrpersonen stehen vor der Aufgabe, solche Prozesse produktiv zu moderieren.

\section{Auffassungsgabe und Geschicklichkeit erwerben}

Die viel beschworene Hand-Auge-Koordination stellt aus Sicht der Schule ein eher untergeordnetes Potenzial dar, auch wenn sich diesbezügliche Studien mittlerweile häufen (vgl. z. B. Andrews \& Murphy, 2006). Gamerinnen und Gamer verfügen offenbar über eine schnellere Auffassungsgabe im visuellen Kanal, ein besseres räumliches Vorstellungsvermögen und diesbezügliches Reaktionsvermögen sowie eine höhere Konzentrationsfähigkeit. In gewissen Bereichen kann dies vor Vorteil sein. Verschiedene Studien kommen z. B. zum Ergebnis, dass Mediziner/innen, die regelmässig Videospiele spielen, mit grösserer Sicherheit laparoskopische (d. h. minimal invasive) Eingriffe am Bildschirm durchführen können (vgl. Rosser et al., 2007). Für die Schule sind solche Potenziale jedoch allzu weit von den Anforderungen des Lehrplans entfernt. Die hohen Geschicklichkeitsanforderungen vieler digitaler Spiele stellen für den schulischen Einsatz eher eine Hürde dar, da das Erlernen der Spielmechanismen allzu viel Unterrichtszeit in Anspruch nehmen würde. Die Lösung für dieses Problem liegt entweder in der Auswahl geeigneter, einfach zu handhabender Spiele oder im Vertrauen auf die oft unterschätzten Fähigkeiten der Schülerinnen und Schüler, ihre in der Freizeit erworbenen Fähigkeiten auch in der Schule zu nutzen. In wenigen Themenbereichen kann jedoch auch die Einübung von Auffassungsgabe Sinn machen, z. B. in Spielen zur Verkehrserziehung 
oder in sportbezogenen Spielen, in denen die Logik bestimmter Spielzüge im Vordergrund steht.

\section{Die Auswahl geeigneter Spiele für den Unterricht}

Zur Realisierung der oben aufgeführten Potenziale können sowohl unterhaltungsorientierte Games als auch spezifische Lernspiele eingesetzt werden, deren Grenzen zu allgemeiner Lernsoftware jedoch fliessend sind. Zum Beginn eines gezielten Einsatzes stehen Lehrpersonen vor der Aufgabe, das Spiel auf seine Eignung hin zu beurteilen. Diese mediendidaktische Analyse umfasst im Idealfall einerseits die didaktischen Potenziale des Spiels (vgl. v. a. Gebel, 2006) und andererseits deren Rolle im geplanten Unterrichtsarrangement (vgl. v. a. Kerres, 2000). Unabhängig davon, ob es sich um ein Lernspiel oder ein unterhaltungsorientiertes Produkt handelt, lassen sich ausgehend von den geschilderten Potenzialen folgende Kriterien als Checkliste für geeignete Spiele formulieren.

- Explorierbarkeit der Inhalte/Zusammenhänge (problemlösend, simulationsähnlich)

- Sinnvoll situierte/strukturierte Spielstory (unterrichtsrelevanter Realitätsbezug, episodisch)

- Motivationspotenzial (aktivierend, selbstwirksamkeitsförderlich, grafisch ansprechend)

- Positives Verhältnis relevanter / irrelevanter Aspekte (verständnisfördernd multimedial)

- Möglichkeiten für Partner/Gruppenarbeit (turn-basiert, Pause-Funktion, Multiplayer-Funktion)

- Schnelle Erlernbarkeit der Spielmechanismen

- Altersangemessenheit und ethische Vertretbarkeit

- Geringe Kosten und Hardware-Anforderungen

Spezifische Lernspiele sind auf die Vermittlung eines bestimmten Inhaltes zugeschnitten und lassen sich vor dem Hintergrund der Checkliste folgendermassen beurteilen (vgl. z. B. Fehr, Heinz, Hieb \& Bauer, 2007). Viele Angebote haben eine bestimmte Altersstufe als Zielgruppe und lassen sich relativ eindeutig einem schulischen Fach zuordnen, so dass der Bezug zum Lehrplan verhältnismässig einfach herzustellen ist. Die Abgrenzung von Lernspielen zu anderer Lernsoftware ist fliessend. Im Internet finden sich verschiedene Datenbanken zur Auswahl geeigneter Produkte. ${ }^{1}$ Die Preise sind mehrheitlich vertretbar und die Hardware-Anforderungen moderat. Teilweise werden diese Programme auch frei über das Internet

www.zum.de, www.evasoft.educa.ch oder www.unterrichtsmedien.ch 
angeboten. Die Problematik solcher Lernspiele im Vergleich zu unterhaltungsbezogenen Spielen liegt jedoch darin, dass die Produkte ein deutlich geringeres Entwicklungsbudget aufweisen und insofern in vielen Bereichen nicht mit kommerziellen Entertainment-Games konkurrieren können. Viele Lernspiele besitzen eine durchsichtige pädagogische Prägung, langweilige repetitive Aufgaben mit stereotypem Feedback und ohne erkennbare Adaptivität. Die Spielehandlung ist linear und einseitig, die Aufgaben wenig komplex und die Grafik unprofessionell. Natürlich gibt es Ausnahmen, wie sie gegenwärtig weltweit unter dem Stichwort "serious games» entwickelt werden. ${ }^{2}$ Erst wenige solcher Spiele finden sich jedoch bisher in deutscher Sprache.

Unterhaltungsorientierte Computerspiele eignen sich ebenfalls in vielen Fällen als Unterrichtsmedien. Sie enthalten bei näherem Hinsehen diverse Aspekte, die als Lerngelegenheiten für Arbeitsaufträge im Unterricht genommen werden können (vgl. Gebel, Gurt \& Wagner, 2005). ${ }^{3}$ Der Unterrichtsbezug ist jedoch, insbesondere wenn der Realitätsbezug nicht auf der Hand liegt, keineswegs einfach herzustellen und die Auswahl bleibt aufwändig. Bis hier ein ausreichender Fundus an ausgearbeiteten Unterrichtsszenarien mit ergänzendem Material in Form von Arbeitsblättern etc. vorliegt, ist eine besondere Eigenleistung von Lehrpersonen gefragt. Der Vorbereitungsaufwand ist hoch, da Lehrpersonen die Spiele selbst gespielt haben müssen, um sinnvolle Aufträge formulieren und Hilfestellungen geben zu können. Ein weiteres Problem ist der oft vorhandene Gewaltaspekt vieler Computerspiele, wobei im Minimum die entsprechende Jugendfreigabe berücksichtigt werden sollte. Die hohen Kosten kommerzieller Spiele erschweren es Schulen, mehrere Lizenzen, geschweige denn einen ganzen Klassensatz aktueller Spiele anzuschaffen. Unzureichende Computerhardware verunmöglicht den Einsatz grafisch intensiver Spiele auf den oft nicht allerneusten Schulcomputern. Besonders vielversprechend sind demgegenüber vor allem Flash-Games und Mini-Games, die mit einem entsprechenden Multimedia-Plugin direkt im Webbrowser laufen und keine Installation erforderlich machen. Diese Art von Spielen entwickelt sich gegenwärtig zu einem ganz eigenen Spielgenre, das sich von den technisch immer aufwändigeren Computer- und Konsolenspielen dezidiert abgrenzt und durch frische und zugleich einfache Grafik und neue Spielprinzipien überzeugt. Auch freie oder Open-sourceSpiele bieten eine gute Option.

\section{Ein Plädoyer für Implementationsforschung in Unterrichtskontexten}

Während Gegnerinnen und Gegner sich auf vermeintlich sichere Fakten aus psychologischen und neurologischen Laborexperimenten, Querschnitts- und Längs-

\footnotetext{
Sammlungen solcher Spiele finden sich z. B. bei www.seriousgames.org; www.socialimpactgames. com; www.watercoolergames.org; www.educationarcade.org oder www.nobelprize.org.

3 Ideen z. B. unter www.spielbar.de; www.bupp.at
} 
schnittuntersuchungen sowie auf die Evidenz bestürzender Einzelfälle stützen, argumentieren die Befürworterinnen und Befürworter, insbesondere im deutschsprachigen Raum, noch allzu häufig im Konjunktiv. Videospiele «können» unter Umständen lernförderliche Potenziale besitzen. In dieser vorsichtigen Formulierung spiegelt sich ein konstruktivistisches Bildungs- und Entwicklungsverständnis und das daraus abgeleitete "Technologiedefizit der Pädagogik» (Luhmann \& Schorr, 1979). Diese Haltung ermöglicht zwar auch eine fundamentale Kritik an den vorschnellen Kausalschlüssen der Gegnerschaft, darüber hinaus bleibt sie jedoch letztlich defensiv. Nötig wäre statt dessen eine verstärkte Orientierung am «Ausprobieren» der postulierten Potenziale in Bildungskontexten. Dies kann einerseits in qualitativen Fallstudien geschehen oder in quantitativen quasi-experimentellen Settings. Aus dem englischsprachigen Raum liegen schon heute eine ganze Reihe von Studien vor, die etwas mehr Selbstbewusstsein bei der Formulierung der Möglichkeiten zulassen würden. Die Meta-Analyse von Vogel et al. (2006) kam auf Basis von 32 kontrollierten Studien, die systematisch ermittelt wurden, zu der zusammenfassenden Aussage, dass beim Lernen mit Games und Simulationen signifikant bessere Leistungen und signifikant positivere Einstellungen zum Lernen zu beobachten sind als bei den Lernenden der Kontrollgruppen, die in traditioneller Weise unterrichtet wurden. Besonders hoch waren die gemessenen Lerngewinne bei Programmen mit grossen Freiheitsgraden und Lernarrangements, die auf ein experimentierendes Lernen abzielen. Solche Befunde werden gestützt von weiteren Metaanalysen zum Lernen mit Multimedia und computerbasierten Simulationen, die ebenfalls stabile positive Effekte ausweisen (z. B. Höffler, 2007; Waxmann \& Connell, 2002; Lee, 1999). Solche Befunde widersprechen nicht grundsätzlich dem genannten Technologiedefizit. Reflektierte Sozialwissenschaft ist sich heute auch in quantitativer Ausrichtung ihrer Grenzen als probabilistischer Disziplin bewusst. Der Nachweis eindimensionaler Korrelationen oder Mittelwertsunterschiede wird zunehmend abgelöst von komplexen Pfadmodellen und Mehrebenenanalysen, die simple Aussagen und vorschnelle Kausalitäten verunmöglichen. Unter dieser Massgabe muss es in praktischen und empirisch begleiteten Versuchen in den nächsten Jahren verstärkt um die Frage gehen, unter welchem Zusammenspiel von Bedingungen Computer- und Videospiele ihre Potenziale entfalten. Qualitative und quantitative Forschung zu den positiven Potenzialen von Videospielen hat gegenüber der Frage nach verschiedenen negativen Auswirkungen einige Jahre Nachholbedarf. Dass für Computer- und Videospiele vielfältige Kompetenzen gefragt sind, dem können bereits Kinder zustimmen (vgl. Fromme, 2000). Mit brauchbaren Spielen in Kombination mit anregenden Unterrichtsideen sind vielleicht auch die Lehrpersonen zu überzeugen. 


\section{Literatur}

Aldrich, Clark. (2004). Simulations and the future of learning. An innovative (and perhaps revolutionary) approach to e-learning. San Francisco: Pfeiffer.

Ammann, Daniel; Hermann, Thomas. (2004). Klicken, lesen und spielend lernen. Interaktive Spielgeschichten für Kinder. Zürich: Verlag Pestalozzianum.

Andrews, Glenda; Murphy, Karen. (2006). Does Video-Game Playing Improve Executive Function? In: M. A. Vanchevsky (Hrsg.), Frontiers in Cognitive Psychology (S. 145-161). Hauppauge, NY: Nova Science Publishers.

Barras, J.-L.; Petko, D. (2007). Computer und Internet in Schweizer Schulen. Bestandsaufnahme und Entwicklung von 2001 bis 2007. In: B. Hotz-Hart (Hrsg.), ICT und Bildung: Hype oder Umbruch? Beurteilung der Initiative Public Private Partnership - Schule im Netz (S. 77-133). Bern: SFIB.

Bauerlein, Mark. (2008). The dumbest generation. How the digital age stupefies young Americains and jeopardizes our future (or, don't trust anyone under 30). New York: Jeremy P. Tarcher/Penguin.

Bransford, John D.; Sherwood, Robert D..; Hasselbring, Ted S.; Kinzer, Charles K.; Williams, Susan M. (1990). Anchored instruction: Why we need it and how technology can help. In: D. Nix; R. Spiro (Hrsg.), Cognition, education and multimedia: Exploring ideas in high technology (S. 115-141). Hillsdale, NJ: Lawrence Erlbaum Associates.

Chandler, Paul; Sweller, John. (1991). Cognitive load theory and the format of instruction. Cognition and Instruction, 8, 293-332.

Cognition and Technology Group at Vanderbilt. (1997). The Jasper Project. Lessons in curriculum, instruction, assessment, and professional development. Mahwah, NJ: Lawrence Erlbaum Associates.

Collins, Allan; Brown, John S.; Newman, Susan E. (1989). Cognitive apprenticeship: teaching the crafts of reading, writing and mathematics. In: L. B. Resnick (Hrsg.), Knowing, learning, and instruction (S. 453-494). Hillsdale, NJ: Lawrence Erlbaum.

Czíkszentmihályi, Mihály. (1990). Flow: the psychology of optimal experience. New York: Harper \& Row.

de Jong, Ton; van Joolingen, Wouter R. (1998). Scientific discovery learning with computer simulations of conceptual domains. Review of Educational Research, 179-202.

Deci, Richard M.; Ryan, Edward L. (1985). Intrinsic Motivation and Self-Determination in Human Behavior. New York: Plenum.

Deci, Richard M.; Ryan, Edward L. (1993). Die Selbstbestimmungstheorie der Motivation und ihre Bedeutung für die Pädagogik. Zeitschrift für Pädagogik, 39(2), 223-238.

Dochy, Filip; Segers, Mien; Van den Bossche, Piet; Gijbels, David. (2003). Effects of problembased learning: a meta-analysis. Learning and Instruction, 13, 533-568.

Egenfeldt-Nielsen, Simon. (2004). Practical barriers in using educational computer games. On the Horizon, 12(1), 18-21.

Fehr, Wolfgang; Heinz, Daniel; Hieb, Markus; Bauer, Matthias. (2007). Spiel- \& Lernsoftware. Pädagogisch beurteilt. Band 17. Online: www.jukobox.de/ jugendamt [25.06. 2008].

Feierabend, Sabine; Rathgeb, Thomas. (2007a). JIM 2007. Jugend, Information, (Multi-)Media. Basisstudie zum Medienumgang 12- bis 19-Jähriger in Deutschland. Medienpädagogischer Forschungsverbund Südwest. Online: http://www.mpfs.de/studien/jim [25.06. 2008].

Feierabend, Sabine; Rathgeb, Thomas. (2007b). KIM-Studie 2006. Kinder und Medien. Computer und Internet. Basisuntersuchung zum Medienumgang 6- bis 13-Jähriger. Medien- 
pädagogischer Forschungsverbund Südwest. Online: http://www.mpfs.de/studien/kim/ [25.06. 2008].

Fischer, Frank. (2001). Gemeinsame Wissenskonstruktion - theoretische und methodologische Aspekte. München: Ludwig-Maximilians-Universität.

Flitner, Andreas. (2004). Spielen - Lernen. Praxis und Deutung des Kinderspiels (11. Aufl.). Weinheim: Beltz.

Fritz, Jürgen. (1995). Warum Computerspiele faszinieren. Empirische Annäherungen an Nutzung und Wirkung von Bildschirmspielen. Weinheim etc.: Juventa.

Fritz, Jürgen. (2003). Warum eigentlich spielt jemand Computerspiele. Macht, Herrschaft und Kontrolle faszinieren und motivieren. In: J. Fritz; W. Fehr (Hrsg.), Computerspiele. Virtuelle Spiel- und Lernwelten (S. 10-24). Bonn: Bundeszentrale für politische Bildung.

Fromme, Johannes. (2000). Die Kompetenzanforderungen der Bildschirmspiele aus der Sicht der Kinder. In: J. Fromme, N. Meder; N. Vollmer (Hrsg.), Computerspiele in der Kinderkultur (S. 97-127). Opladen: Leske+Budrich.

Gebel, Christa. (2006). Kompetenzförderliche Potenziale unterhaltender Computerspiele. Unterrichtswissenschaft, 34(4), 290-309.

Gebel, Christa; Gurt, Michael; Wagner, Ulrike. (2005). Kompetenzförderliche Potenziale populärer Computerspiele. Online: http://www.abwf.de/content/main/publik/report/ 2005/report-92b.pdf [25.06. 2008].

Gee, James Paul. (2003). What video games have to teach us about learning and literacy. Basingstoke: Palgrave Macmillan.

Grüsser, Sabine M.; Thalemann, Ralf. (2006). Computerspielsüchtig? Rat und Hilfe. Bern: Huber.

Hepp, Andreas; Vogelgesang, Waldemar. (2008). Die LAN-Szene. Vergemeinschaftungsformen und Aneignungsweisen. In: T. Quandt, J. Wimmer; J. Wolling (Hrsg.), Die Computerspieler. Studien zur Nutzung von Computergames (S. 97-112). Wiesbaden: VS Verlag für Sozialwissenschaften.

Höffler, Tim Niclas. (2007). Lernen mit dynamischen Visualisierungen: Metaanalyse und experimentelle Untersuchungen zu einem naturwissenschaftlichen Lerninhalt. Elektronische Dissertation der Universität Duisburg-Essen. Online: http://duepublico.uni-duisburg-essen.de/ [25.06. 2008].

Jansz, Jeroen; Martens, Lonneke. (2005). Gaming at a LAN event: the social context of playing video games. New Media \& Society, 7(3), 333-355.

Johnson, David W.; Johnson, Roger T. (1998). Cooperative Learning And Social Interdependence Theory. Online: http://www.co-operation.org/pages/SIT.html [25.06. 2008].

Johnson, Steven. (2005). Everything bad is good for you. How popular culture is making us smarter. London: Allen Lane.

Kerres, Michael. (2000). Mediendidaktische Analyse digitaler Medien im Unterricht. Computer und Unterricht, 10(1), 26-28.

Kirriemuir, John; McFarlane, Angela. (2003). Literature Review in Games and Learning. Online: http://www.nestafuturelab.org/research/lit_reviews.htm [01.02. 2006].

Klimmt, Christoph. (2004). Computer- und Videospiele. In: R. Mangold, P. Vorderer; G. Bente (Hrsg.), Lehrbuch der Medienpsychologie (S. 695-716). Göttingen: Hogrefe.

Klimmt, Christoph; Hartmann, Thilo. (2006). Effectance, Self-Efficacy, and the Motivation to Play Video Games. In: P. Vorderer; J. Bryant (Hrsg.), Playing Video Games. Motives, Responses, and Consequences (S. 132-145). Mahwah: Lawrence Erlbaum Associates. 
Krützer, B.; Probst, H. (2006). IT-Ausstattung der allgemein bildenden und berufsbildenden Schulen in Deutschland. Bestandsaufnahme 2006 und Entwicklung 2001 bis 2006. Bonn und Berlin: Bundesministerium für Bildung und Forschung (BMBF).

Kutner, Lawrence; Olson, Cheryl K. (2008). Grand Theft Childhood. The Surprising Truth About Violent Video Games. And What Parents Can Do. New York: Simon \& Schuster.

Lave, Jean; Wenger, Etienne. (1991). Situated learning: Legitimate peripheral participation. Cambridge: Cambridge University Press.

Lee, June. (1999). Effectiveness of computer-based instructional simulation: A meta-analysis. International Journal of Instructional Media, 26, 71-85.

Lee, Kwan Min; Peng, Wei. (2006). What Do We Know About Social and Psychological Effects of Computer Games? A Comprehensive Review of Current Literature. In: P. Vorderer; J. Bryant (Hrsg.), Playing Video Games. Motives, Responses, and Consequences (S. 325-346). Mahwah: Lawrence Erlbaum Associates.

Luhmann, Niklas; Schorr, Karl Eberhard. (1979). Das Technologiedefizit der Erziehung und die Pädagogik. Zeitschrift für Pädagogik, 25(3), 315-345.

Mayer, Richard E. (2001). Multimedia Learning. Cambridge: Cambridge University Press.

Mitchell, Alice; Savill-Smith, Carol. (2004). The use of computer and video games for learning. A review of the literature. London: Learning and Skills Development Agency.

Oerter, Rolf. (1999). Psychologie des Spiels. Ein handlungstheoretischer Ansatz (Durchges. Neuausg.). Weinheim: Beltz.

Petko, Dominik; Reusser, Kurt. (2005). Das Potential von interaktiven Lernressourcen zur Förderung von Lernprozessen. In: D. Miller (Hrsg.), eLearning. Eine multiperspektivische Standortbestimmung (S. 161-185). Bern: Haupt.

Pfeiffer, Christian; Mössle, Thomas; Kleimann, Matthias; Rehbein, Florian. (2007). Die PlSA-Verlierer - Opfer ihres Medienkonsums. Eine Analyse auf der Basis verschiedener empirischer Untersuchungen. Kriminologisches Forschungsinstitut Niedersachsen e.V. Online: http://www.kfn.de/versions/kfn/assets/pisaverlierer.pdf [25.06. 2008].

Preckel, Daniel. (2004). Problembasiertes Lernen: Löst es die Probleme der traditionellen Instruktion? Unterrichtswissenschaft, 32(4), 274-287.

Prensky, Marc. (2001). Digital game-based learning. St. Paul, MN: Paragon House.

Reeves, Byron; Malone, Thomas et al. (2007). Leadership in Games and at Work: Implications for the Enterprise of Massively Multiplayer Online Role-playing Games. Report prepared for IBM. Online: http://www.seriosity.com/downloads/Leadership_In:_Games_ Seriosity_and_IBM.pdf [25.06. 2008].

Reinmann-Rothmeier, Gabi; Mandl, Heinz. (2006). Unterrichten und Lernumgebungen gestalten. In: A. Krapp; B. Weidenmann (Hrsg.), Pädagogische Psychologie. Ein Lehrbuch (5. vollst. überarb. Aufl. ed., S. 613-658). Weinheim: BeltzPVU.

Reusser, Kurt. (2005). Problemorientiertes Lernen - Tiefenstruktur, Gestaltungsformen, Wirkung. Beiträge zur Lehrerbildung, 23(2), 159-182.

Rosser, James C.; Lynch, Paul J.; Cuddihy, Laurie; Gentile, Douglas A.; Klonsky, Jonathan; Merrell, Ronald. (2007). The Impact of Video Games on Training Surgeons in the 21st Century. Archives of Surgery, 142(2), 181-186.

Salomon, Gavriel. (1983). The differential investment of mental effort in learning from differenz sources. Educational Psychologist, 18(1), 42-50.

Salomon, Gavriel. (1984). Television is «easy» and print is «tough»: The differential investment of mental effort in learning as a function of perceptions and attributions. Journal of Educational Psychology, 74(4), 647-658. 
Sandford, Richard; Williamson, Ben. (2005). Games and learning. A handbook from NESTA Futurelab. Online: http://www.nestafuturelab.org/research/handbooks.htm [25.06. 2008].

Schnotz, Wolfgang. (2002). Wissenserwerb mit Texten, Bildern und Diagrammen. In: L. J. Issing; P. Klimsa (Hrsg.), Information und Lernen mit Multimedia und Internet. Lehrbuch für Studium und Praxis (3., vollst. überarb. Aufl. ed., S. 65-81). Weinheim: Beltz PVU.

Schrackmann, I.,Knüsel, D.,Moser, T.,Mitzlaff, H.; Petko, D. (2008). Computer und Internet in der Primarschule. Theorie und Praxis von ICT im Unterricht mit 20 Videobeispielen auf zwei DVDs. Oberentfelden / Aarau: Sauerländer

Slavin, Robert E. (1996). Research on Cooperative Learning and Achievement: What We Know, What We Need to Know. Contemporary Educational Psychology, 21(1), 43-69.

Spitzer, Manfred. (2005). Vorsicht Bildschirm! Elektronische Medien, Gehirnentwicklung, Gesundheit und Gesellschaft. Stuttgart: Klett.

Squire, Kurt. (2003). Video Games in Education. Online: http://www.cyberfest.us/Education/ Video_Games_in_Education-MIT_Study.pdf [25.06. 2008].

Susi, Tarja; Johannesson, Mikael; Backlung, Per. (2007). Serious Games - An Overview. Online: http://www.his.se/upload/19354/HS-\%20IKI\%20-TR-07-001.pdf [25.06. 2008].

Van Eck, Richard. (2006). Digital Game-Based Learning. It's Not Just the Digital Natives Who Are Restless. EDUCAUSreview, 2006(March/April), 16-30.

Vogel, Jennifer J.; Vogel, David S.; Cannon-Bowers, Jan; Bowers, Clint a.; Muse, Kathryn; Wright, Michelle. (2006). Computer Gaming and Interactive Simulations for Learning: A Meta Analysis. Journal for Educational Computing Research, 34(3), 229-243.

Waxmann, Hersh C.; Connell, Michael L. (2002). A Quantitative Synthesis of Recent Research on the Effects of Teaching and Learning With Technology on Student Outcomes. Online: http://www.ncrel.org/tech/effects2/waxman.pdf [25.06. 2008].

Weidenmann, Bernd. (2002). Multicodierung und Multimodalität im Lernprozess. In: L. J. Issing; P. Klimsa (Hrsg.), Information und Lernen mit Multimedia und Internet (3. Aufl. ed., S. 45-62). Weinheim: Beltz PVU.

Wünsch, Carsten; Jenderek, Bastian. (2008). Computerspielen als Unterhaltung. In: T. Quandt, J. Wimmer; J. Wolling (Hrsg.), Die Computerspieler. Studien zur Nutzung von Videogames (S. 41-56). Wiesbaden: VS Verlag für Sozialwissenschaften. 